\title{
Economic evaluation of the specialized donor care facility for thoracic organ donor management
}

\author{
Jason M. Gauthier ${ }^{1}$, Maria B. Majella Doyle ${ }^{2}$, William C. Chapman ${ }^{2}$, Gary Marklin ${ }^{3}$, Chad A. Witt ${ }^{4}$, \\ Elbert P. Trulock ${ }^{4}$, Derek E. Byers ${ }^{4}$, Ramsey R. Hachem ${ }^{4}$, Michael K. Pasque ${ }^{1}$, Bryan F. Meyers ${ }^{1}$, \\ G. Alexander Patterson ${ }^{1}$, Ruben G. Nava ${ }^{1}$, Benjamin D. Kozower ${ }^{1}$, Daniel Kreisel ${ }^{1,5}$, Su-Hsin Chang ${ }^{6}$, \\ Varun Puri ${ }^{1}$
}

${ }^{1}$ Division of Cardiothoracic Surgery, ${ }^{2}$ Division of Abdominal Organ Transplantation, Department of Surgery, ${ }^{3}$ Mid-America Transplant, ${ }^{4}$ Division of Pulmonary and Critical Care Medicine, Department of Medicine, ${ }^{5}$ Department of Pathology and Immunology, ${ }^{6}$ Division of Public Health Sciences, Department of Surgery, Washington University School of Medicine, Saint Louis, MO, USA

Contributions: (I) Conception and design: JM Gauthier, MBM Doyle, WC Chapman, G Marklin, D Kreisel, SH Chang, V Puri; (II) Administrative support: G Marklin, D Kreisel, SH Chang, V Puri; (III) Provision of study materials or patients: JM Gauthier, G Marklin, SH Chang; (IV) Collection and assembly of data: JM Gauthier, G Marklin, SH Chang, V Puri; (V) Data analysis and interpretation: JM Gauthier, MBM Doyle, WC Chapman, G Marklin, MK Pasque, D Kreisel, BD Kozower, SH Chang, V Puri; (VI) Manuscript writing: All authors; (VII) Final approval of manuscript: All authors.

Correspondence to: Jason M. Gauthier, MD. Division of Cardiothoracic Surgery, Washington University School of Medicine, Campus Box 8109 , 660 South Euclid Avenue, St. Louis, MO 63110, USA. Email: gauthier.jason.m@wustl.edu.

Background: Over the last decade two alternative models of donor care have emerged in the United States: the conventional model, whereby donors are managed at the hospital where brain death occurs, and the specialized donor care facility (SDCF), in which brain dead donors are transferred to a SDCF for medical optimization and organ procurement. Despite increasing use of the SDCF model, its cost-effectiveness in comparison to the conventional model remains unknown.

Methods: We performed an economic evaluation of the SDCF and conventional model of donor care from the perspective of U.S. transplant centers over a 2-year study period. In this analysis, we utilized nationwide data from the Scientific Registry of Transplant Recipients and controlled for donor characteristics and patterns of organ sharing across the nation's organ procurement organizations (OPOs). Subgroup analysis was performed to determine the impact of the SDCF model on thoracic organ transplants.

Results: A total of 38,944 organ transplants were performed in the U.S. during the study period from 13,539 donors with an observed total organ cost of $\$ 1.36$ billion. If every OPO assumed the cost and effectiveness of the SDCF model, a predicted 39,155 organ transplants (+211) would have been performed with a predicted total organ cost of $\$ 1.26$ billion (-\$100 million). Subgroup analysis of thoracic organs revealed that the SDCF model would lead to a predicted 156 additional transplants with a cost saving of $\$ 24.6$ million.

Conclusions: The U.S. SDCF model may be a less costly and more effective means of multi-organ donor management, particularly for thoracic organ donors, compared to the conventional hospital-based model.

Keywords: Organ donor management; transplantation, heart; transplantation; lung

Submitted Apr 04, 2020. Accepted for publication Aug 15, 2020.

doi: $10.21037 /$ jtd-20-1575

View this article at: http://dx.doi.org/10.21037/jtd-20-1575 


\section{Introduction}

The number of deceased organ donors and solid organ transplants in the United States (U.S.) has consistently risen in recent years, with approximately 36,500 transplants from 10,700 deceased donors performed in 2018 alone (1). The current method of organ allocation allows donors to be matched with appropriate recipients nationwide, requiring substantial coordination and typically necessitating travel by several transplant teams for each donor (2). Donor management, organ allocation, and procurement operations are coordinated by 58 federally mandated, non-profit organ procurement organizations (OPOs), each managing donors within its respective geographic service area. Traditionally, this requires a donor's OPO to communicate extensively with multiple transplant centers and coordinate care with the referring hospital where the donor resides. In an effort to optimize the efficiency of this process and potentially reduce costs, our local OPO, Mid-America Transplant (MOMA), built the country's first freestanding specialized donor care facility (SDCF) in 2001. In this model, donation after brain death donors are transferred from the referring hospital to the SDCF for medical optimization while organ allocation is coordinated with transplant centers (Figure 1). The SDCF maintains its own in-house critical care unit, laboratory, radiographic equipment (i.e., CT scanner), and procurement operating rooms, resulting in a streamlined process of donor care. After the donor workup is complete and organs have been accepted by transplant centers, procurement teams travel to the SDCF for organ procurement.

By 2011, 93\% of all procurements from MOMA were being conducted at the SDCF, which was associated with a reduction in surgeon air travel and overall cost of donor management $(3,4)$. Furthermore, organ procurement from MOMA's SDCF has been associated with an increase in organ yield per donor $(4,5)$. At least 12 other OPOs in the U.S. have since either adopted the SDCF model or are in the process of doing so. However, a national comparison of the SDCF model with the conventional model of donor management while controlling for donor characteristics has not been performed. Furthermore, a nationwide economic evaluation from the perspective of U.S. transplant centers has also not been previously conducted. This holds tremendous importance with regards to donor management policy given the limited resources and wide variability of donor characteristics and organ acquisition costs. Not only is this important for transplant centers in the U.S., it is likely that the SDCF model of care has important characteristics that can be successfully adapted to support organ transplantation, particularly for thoracic organs, worldwide.

Given the sustained increase in organ donors over the last decade and the high cost associated with organ acquisition, it is critically important to compare the various models of donor care. To address this gap, we conducted an economic evaluation of the SDCF model compared to the conventional model of donor care in the U.S. from the perspective of transplant centers. We present the following article in accordance with the Consolidated Health Economic Evaluation Reporting Standards (CHEERS) reporting checklist (available at http://dx.doi.org/10.21037/ jtd-20-1575).

\section{Methods}

In this analysis we conducted an economic evaluation from a transplant center's perspective to compare the SDCF model with the conventional model of hospital-based donor care. To accomplish this, we compare the observed (actual) cost and effectiveness of the nation's OPOs during the study period with that of a predicted scenario in which all OPOs adopted the SDCF model of care. We chose to use nationwide data from the Scientific Registry of Transplant Recipients (SRTR) (6) to measure effectiveness, as this data is rigorously adjusted for individual donor characteristics and allows for accurate comparisons of performance among our country's OPOs. We adjusted for patterns of organ sharing across the nation's OPOs which vary across different regions of the country.

Organ acquisition charge (OAC) was used to compute organ costs. When a transplant center (e.g., Barnes-Jewish Hospital) accepts an organ for transplantation, the OAC is paid by that center to the respective OPO (e.g., MOMA) and ultimately reimbursed by private insurance companies or the government via the Center for Medicare \& Medicaid Services. The OAC is used by OPOs to fund the cost of managing an organ donor in the hospital, coordinating the donation process, reimbursing hospitals for operating room charges, and in the case of an SDCF, transportation and management of the donor at the facility. Therefore, the OAC of an organ represents the unit cost of that organ to a transplant center. At MOMA, the OAC for a given organ does not change based on donation after brain death or cardiac death status. 


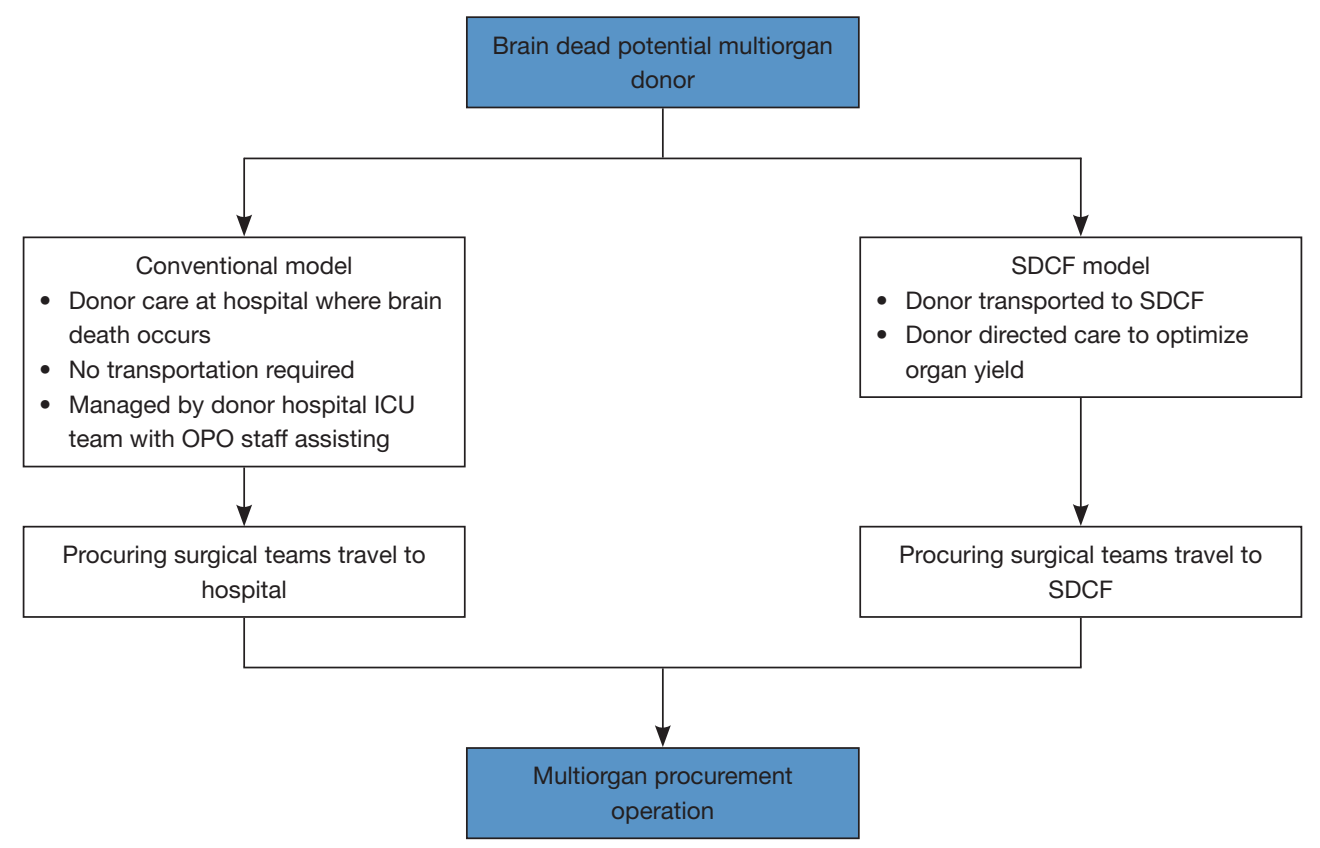

Figure 1 Illustration of the specialized donor care facility (SDCF) model of donor care. In the conventional model of hospital-based donor care, a referring hospital notifies an organ procurement organization (OPO) of a potential organ donor, and the OPO then coordinates donor care and workup between the referring hospital, OPO, and transplant centers. In the SDCF model, the OPO transports brain dead donors to the SDCF for workup, medical optimization, recipient matching, and coordination of procurement, thereby streamlining donor care.

Cost savings in the absence of comparable or improved effectiveness would be undesirable in any new model of care. Therefore, effectiveness was measured using each OPO's observed and predicted number of transplants performed during the study period for heart, lung, liver, kidney, and pancreas. Single and double lung transplants were counted as one organ, while kidney transplants were counted separately. Due to their rarity, small intestine transplants were excluded from the analyses (Figure S1). Both donation after cardiac death and brain-dead donors were included in this analysis.

\section{Data}

\section{Effectiveness}

The number of organ donors and the observed and expected number of transplants for each OPO was obtained from OPO-specific reports from the SRTR (Supplementary material, A). The expected number of transplants for each organ is derived from a statistical model that adjusts for 38 donor characteristics, such as age, cause of death, and medical co-morbidities, and is published by the SRTR (6) (see Table S1). The organ-specific observed-to-expected ratio (O:E) for each OPO represents the observed number of transplants for a given organ divided by the expected number of transplants for that organ. Local organ transplants were defined as those donated to a transplant center within the OPO's donation service area (DSA), while export organ transplants were those donated outside of an OPO's DSA (Figure 2). It is important to account for the local and export organ transplants in each OPO as the OAC for export organs is usually higher due to travel costs and greater logistical burden. January 1, 2013 to December 31, 2014 was chosen as the study period, as this was the most recent period for which all required data were available.

\section{Cost}

The local and export organ-specific OACs for each OPO were obtained from OPO-reported data from the year 2014 (presented at the Association of Organ Procurement Organizations annual meeting). The OPO-specific OACs have not been reported publicly since 2014 . 


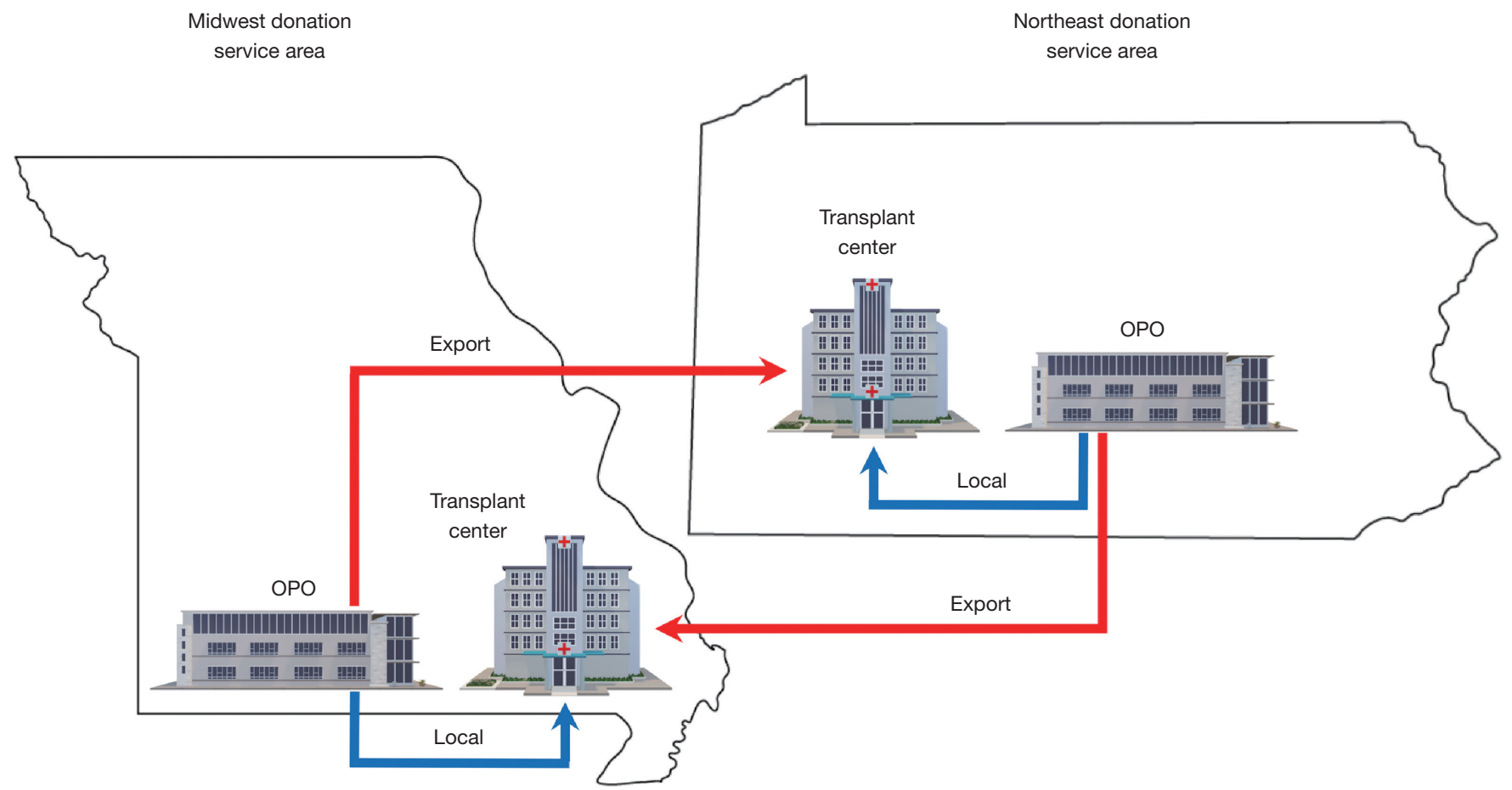

Figure 2 Illustration of local and export donations from two organ procurement organizations (OPOs). Each of the 58 OPOs in the U.S. manages a donation service area (DSA) (represented by black lines), which collectively encompass all 50 states. Using organ allocation algorithms and current policies, an OPO matches donor organs to recipients both inside its DSA, termed "local" transplants (blue lines), and outside its service area, termed "export" transplants (red lines). Export donations generally confer a higher OAC due to increased administrative burden and travel costs. These DSA boundaries are for illustrative purposes only and do not represent actual DSA boundaries.

\section{Observed organ transplants}

For each OPO, the number of organ-specific observed transplants were obtained from the SRTR reports (6). Organ-specific observed total transplants nationwide were obtained by summing observed transplants from each OPO (Supplementary material, B).

\section{Observed costs}

For each OPO, organ-specific local and export OACs were used to compute the organ-specific observed average OAC (a weighted average of local and export OACs with weights determined by the number of local and export organs), and observed total costs (sum of local and export costs) (Supplementary material, C).

As an example, during the study period OPO $A$ (conventional model) observed 307 liver transplants, including 203 local transplants with liver OAC $=\$ 30,000$ and 104 export transplants with liver $\mathrm{OAC}=\$ 36,000$.
Thus, the liver-specific observed total costs for OPO $A=$ $(\$ 30,000 \times 203)+(\$ 36,000 \times 104)=\$ 9,834,000$.

We then computed organ-specific observed average OAC nationwide by averaging observed organ-specific OACs from each OPO. Organ-specific observed total costs nationwide were derived from the sum of observed organspecific total costs from each OPO.

\section{Predicted transplants assuming nationwide adoption of the SDCF model}

We assumed a scenario in which all OPOs in the U.S. adopted the SDCF model and achieved the same effectiveness. Data from MOMA's performance was used to inform effectiveness, as this was the nation's only fully functioning SDCF in the year prior to the study period. Under such a scenario, we assume that each OPO has the same O:E as that of the SDCF while retaining its own capacity (E). Therefore, the organ-specific predicted total 
transplants was calculated by multiplying the organ-specific expected transplants for each OPO by the SDCF's O:E ratio for that organ.

As an example, OPO $A$ (conventional model) observed 70 lung transplants from 367 donors during the study period, while 69.4 lung transplants were expected based on the SRTR's statistical model (6). During the same period, the SDCF's lung O:E was 1.18. Therefore, the predicted number of lung transplants from OPO $A$ based on the SDCF's performance $=69.4 \times 1.18=81.9$. The organ-specific predicted total transplants nationwide were calculated by summing the predicted transplants for each OPO across all OPOs (Supplementary material, D).

\section{Predicted costs assuming nationwide adoption of the SDCF model}

Under the aforementioned scenario in which all OPOs in the U.S. adopted the SDCF model, the observed OAC of the SDCF was assumed to be the organ-specific predicted average OAC for all OPOs (Supplementary material, E). The overall predicted average OAC was obtained by averaging the organ-specific predicted average OACs for the studied organs.

As an example, during the study period OPO $A$ (conventional model) was expected to perform 304 liver transplants with local and export proportions of 0.66 and 0.34 , respectively. The SDCF's OAC for local liver transplants was $\$ 26,000$, OAC for export liver transplants was $\$ 38,000$, and O:E ratio of the SDCF model for liver was 1.06. As described above, the predicted number of liver transplants for OPO $A=304 \times 1.06=322$. Thus, the liver-specific predicted total costs from OPO $A=322 \times$ $(\$ 26,000 \times 0.66+\$ 38,000 \times 0.34)=\$ 9,685,760$.

\section{Statistical and economic analysis}

The primary measure of benefit was the difference in total transplants and cost between the observed and predicted scenarios. The secondary measure of benefit was the difference in organ-specific transplants and cost between the two scenarios. All OPOs lacking complete cost or effectiveness data during the study period were excluded from the analysis. Descriptive statistics were computed. Bootstraps were performed to resample OPOs 1,000 times with replacement when computing the predicted transplants and costs to obtain means and $95 \%$ confidence intervals (CIs). Cost-effectiveness plane was plotted to demonstrate effectiveness (mean overall predicted total transplants from the bootstraps and overall observed total transplants) against cost (mean overall predicted total cost from the bootstraps and overall observed total cost). All analyses were conducted using Microsoft Excel, version 16 (Albuquerque, NM, USA).

\section{Results}

\section{Nationwide observed cost and effectiveness}

During the study period MOMA was the only established SDCF in the country which was transferring nearly all of its donors to the freestanding facility. Thirteen OPOs did not have complete data available and were excluded from the analysis (Figure S1). Nationwide, 38,944 transplants of the 5 organs of interest (heart, kidney, liver, lung, and pancreas) were observed from 13,539 individual donors (289 transplants per 100 donors) over the 2-year study period (Table 1).

The nationwide observed total costs paid by transplant centers to the OPOs was $\$ 1.36$ billion (Table 2). The observed average OAC was $\$ 37,599$ per organ. Lungs had the highest observed average OAC $(\$ 46,276)$, followed by heart $(\$ 37,743)$.

\section{Nationwide predicted costs and effectiveness presuming SDCF model of care}

Assuming nationwide adoption of the SDCF model, the overall predicted total transplants nationwide would have been 39,155 (95\% CI: 38,977-39,334), an increase of 211 transplants compared to the observed effectiveness (Table 1). The overall predicted total organ cost nationwide was $\$ 1.26$ billion (95\% CI: $\$ 1.26-1.27$ billion), a decrease of $\$ 100$ million compared to the overall observed total organ costs nationwide. The predicted average OAC for every organ was lower compared to the observed average OAC based on the conventional model of care (overall mean $\$ 32,127 v s$. $\$ 37,599)$ (Table 2).

\section{Evaluation of the cost and effectiveness of nationwide adoption of the SDCF model}

We evaluated the cost and effectiveness of nationwide adoption of the SDCF model by comparing the overall total transplants and organ costs between the observed and predicted scenarios. The predicted scenario dominated 
Table 1 Observed and predicted effectiveness of organ transplantation

\begin{tabular}{|c|c|c|c|c|c|c|}
\hline Number of transplants & HR & $\mathrm{KI}$ & $\mathrm{LI}$ & LU & PA & Overall \\
\hline $\begin{array}{l}\text { Predicted total transplants } \\
\text { nationwide }(95 \% \mathrm{Cl})\end{array}$ & $\begin{array}{c}3,914 \\
(3,895,3,932)\end{array}$ & $\begin{array}{c}19,530 \\
(19,444,19,615)\end{array}$ & $\begin{array}{c}10,670 \\
(10,621,10,719)\end{array}$ & $\begin{array}{c}3,387 \\
(3,369,3,404)\end{array}$ & $\begin{array}{c}1,656 \\
(1,648,1,663)\end{array}$ & $\begin{array}{c}39,155 \\
(38,977,39,334)\end{array}$ \\
\hline $\begin{array}{l}\text { Predicted transplants per } \\
100 \text { donors nationwide }[95 \% \mathrm{Cl}]\end{array}$ & $29[29,29]$ & $145[145,145]$ & $79[79,79]$ & $25[25,25]$ & $13[12,13]$ & $290[290,290]$ \\
\hline
\end{tabular}

Observed transplants refers to the actual number of organ transplants in total and per 100 donors during the study period. Predicted transplants refers to the number of organ transplants in total and per 100 donors based on the SDCF model and includes $95 \%$ confidence intervals (Cls). 95\% confidence intervals were generated by the bootstrapped results. HR, heart; KI, kidney; LI, liver; LU, lung; PA, pancreas; AVG, national average; SDCF, specialized donor care facility.

Table 2 Observed and predicted cost of organ transplantation

\begin{tabular}{|c|c|c|c|c|c|c|}
\hline Cost & HR & $\mathrm{KI}$ & $\mathrm{LI}$ & LU & PA & Overall \\
\hline $\begin{array}{l}\text { Predicted total cost } \\
(95 \% \mathrm{Cl})(\$)\end{array}$ & $\begin{array}{r}126,630,733 \\
(126,031,400 \\
127,230,066)\end{array}$ & $\begin{array}{c}622,847,899 \\
(620,086,201 \\
625,609,598)\end{array}$ & $\begin{array}{c}320,790,524 \\
(319,346,831, \\
322,234,218)\end{array}$ & $\begin{array}{r}137,715,189 \\
(137,040,238 \\
138,390,140)\end{array}$ & $\begin{array}{r}53,464,409 \\
(53,219,362 \\
53,709,456)\end{array}$ & $\begin{array}{c}1,261,448,754 \\
(1,255,724,031 \\
1,267,173,477)\end{array}$ \\
\hline Observed average OAC (\$) & 37,743 & 32,053 & 36,299 & 46,276 & 35,622 & 37,599 \\
\hline
\end{tabular}

Observed total cost and OAC refer to the actual total cost and average OAC during the study period. Predicted total cost and OAC refer to the total cost, including 95\% confidence intervals (Cls), and average OAC based on the SDCF model. OACs are given as the weighted national average. 95\% confidence intervals were generated by the bootstrapped results. All costs are in 2014 U.S. dollars. HR, heart; KI, kidney; LI, liver; LU, lung; PA, pancreas; OAC, organ acquisition charge; SDCF, specialized donor care facility.

the observed scenario by being less costly (i.e., nationwide predicted total costs - observed total costs $=-\$ 100$ million; last column in Table 2) and more effective (i.e., nationwide predicted transplants - observed transplants $=+211$ transplants; last column in Table 1) (Figure 3A).

Among the 211 additional transplants that were predicted with adoption of the SDCF model, 156 (74\%) of these were from thoracic organs (heart and lungs). Subgroup analysis of thoracic organs alone demonstrated that the predicted scenario dominated the observed scenario by leading to an increase in thoracic transplants $(+156)$ at a lower total organ cost (-\$24.6 million) (Figure 3B).

\section{Discussion}

We have performed an economic evaluation comparing the SDCF model of donor care to the conventional model of hospital-based care in the U.S. while accounting for regional variations in donor characteristics and patterns of organ sharing across the country's OPOs. Our results show that the SDCF model may be a cost-effective means of donor management in the U.S. from a transplant center's perspective, which may lead to an increase in thoracic organ transplants at a lower per transplant cost. While the predicted total number of organ transplants was slightly higher than the observed number of organ transplants, the predominant reason for the SDCF model's cost-effectiveness is a substantially lowered predicted organ cost. With the changing landscape of organ donor management and the consistent increase in organ donors in recent years (7), these findings have important and farreaching implications for health care economics and donor management policy (8), both at a national and international level. 

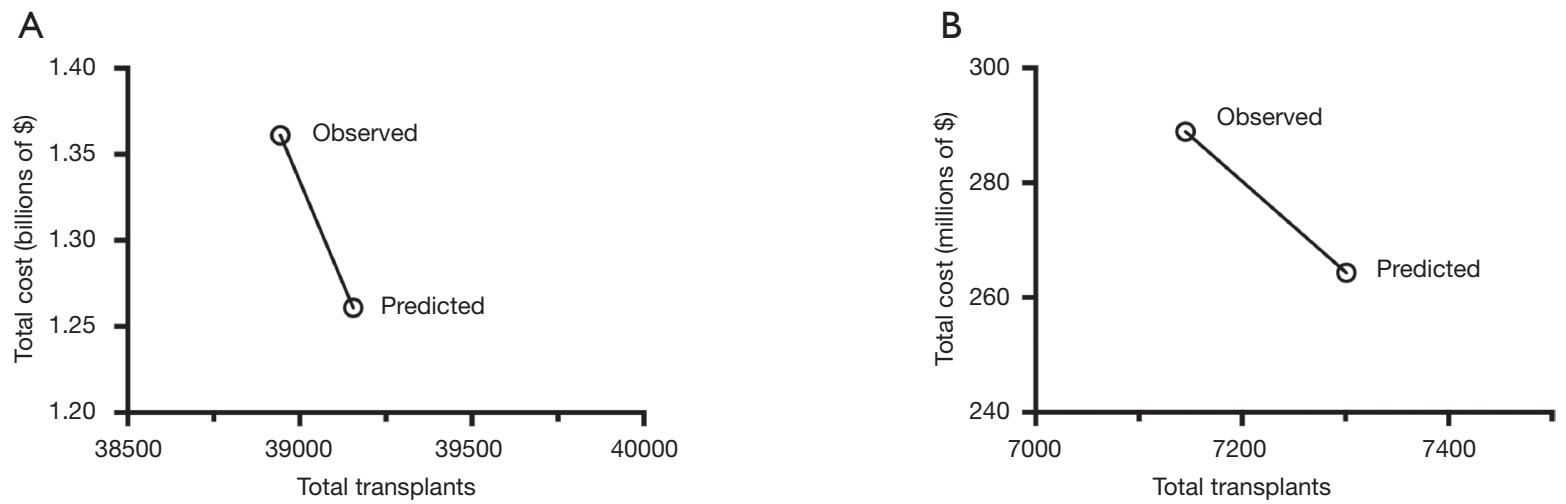

Figure 3 Observed and predicted overall cost and effectiveness of the specialized donor care facility (SDCF) model of care compared to the conventional model. Overall total costs nationwide are shown in billions and millions of U.S. dollars for all organs (A) and thoracic organs (B), respectively. Overall total transplants nationwide refer to the sum of all transplants done by the 45 organ procurement organizations (OPOs) in this study for the 5 organs of interest (heart, liver, lung, kidney, pancreas). Thoracic organs represent the sum of lung and heart transplants alone. The observed data point is based on the actual outcomes during the study period, while the predicted data point is based on nationwide adoption of the SDCF model during the study period.

The reasons for cost savings using the SDCF model are multifactorial but largely appear to be a result of lower overhead costs and greater efficiency in donor management. In the conventional model of donor care, all procedures and hospital-based workup add to the overall cost of donor management, which is paid for by the donor's OPO. For example, if a donor needs a CT scan and bronchoscopy while at the referring hospital, both studies will confer additional cost to the OPO, which will ultimately be passed on to transplant centers through the OAC. Conversely, in the SDCF model the diagnostic workup is centralized, with radiographic studies, diagnostic testing, and laboratory analysis being performed at the SDCF via OPO-owned resources. Similarly, the SDCF model is likely to result in lower professional fees as well. A donor requiring bronchoscopy and dialysis in the conventional setting will be managed by a pulmonologist and a nephrologist, while in the SDCF model, a critical care physician or other personnel, who are extremely familiar with donor management and employed by the OPO, will provide these services. Donor care is a complex process that most hospitals are unfamiliar with. In this regard, the SDCF can be regarded as a "high-volume" center that performs these complex tasks frequently and efficiently. By co-locating services, such as radiographic machines, diagnostic testing equipment, intensive care units, and procurement operating rooms, the SDCF is able to simplify the donor management process. Despite recent changes in organ allocation policy in the U.S., the benefits of the SDCF are likely to be maintained due to inherent efficiencies of the model that facilitates a streamlined organ donor management and recovery process and lowers the cost of donor care.

The SDCF model can reliably institute specific algorithms to increase organ yield, which may be one factor contributing to the increase in effectiveness. A prior study by our group demonstrated that a lung focused resuscitation protocol implemented by a SDCF led to increased organ yield (5). Similarly, a previous study from our institution demonstrated that transferring a donor to a SDCF increased the overall organ yield (3.43 vs. 2.69 organs per donor, $\mathrm{P}<0.0001)$ and was associated with a lower mean donor recovery cost $(\$ 16,153$ vs. $\$ 33,161, \mathrm{P}<0.0001)$ (4). The transfer process itself appears to be relatively quick, with a median time from family consent to transfer of 8.6 hours, and has been associated with a very high family satisfaction (4). We have also found that procurement from the SDCF, rather than a referring hospital, was correlated with higher likelihood of daytime, rather than after-hours transplants (9). Therefore, it appears that transfer to a SDCF is efficient from a number of perspectives, including that of surgeons and donor families, and may confer other advantages not examined in this study. It remains to be seen if the benefits of a SDCF appreciated in the U.S. will be seen in other countries. To our knowledge no similar facilities exist outside of the U.S. However, we strongly anticipate that the fundamental efficacy of the SDCF model 
can be replicated not only in the U.S., but internationally.

Since the SDCF model requires a freestanding organ recovery center, the cost of initiating this model is worthy of discussion. A prior study of our local SDCF estimated the startup cost to be $\$ 5.34$ million (construction, medical equipment, etc.) (4). In the current study we found a cost savings of $\$ 100$ million for 45 OPOs using the SDCF model, resulting in approximately $\$ 2.2$ million in savings per OPO during the study period. Therefore, one could envision that OPOs could use the differences in cost of donor management to offset startup costs. Our results also suggest that implementation of the SDCF model may be in the best interest of transplant centers as well, and they may be incentivized to fund a portion of a SDCF's startup cost.

A few critical elements are required for the SDCF model to succeed and these should be carefully considered before another country or other U.S. OPOs adopt this model. First, the donor volume should be high enough that the cumulative OACs can finance the costs of running a SDCF. The leadership at our local OPO has estimated this number to be around 100 donors per year, which would have encompassed 38 of the 58 OPOs during our study period. It appears that the SDCF model functions optimally when built geographically close to high volume transplant centers, as this has been shown to reduce travel time and the number of "fly outs" by the procurement team in both real (3) and hypothetical (10) models of donor management. In the setting of new U.S. organ allocation policies, which have been shown to increase air travel (8), it may be ideal to locate new SDCFs close to airports.

Our results must be interpreted with some limitations in mind. We have conducted an economic evaluation from the perspective of U.S. transplant centers, which is only one of several perspectives that one could take when conducting such an analysis. The perspective of the payer (i.e., private insurance companies or the Center for Medicare \& Medicaid Services), the referring hospital, and donor families was not examined in this analysis. The study period represents the most recent era for which all required U.S. cost data were available, and costs may have changed since then. Notably, while all 58 OPOs in the country are not for profit organizations, various OPOs are likely to have different margins of "profit" for different organs; unfortunately, this information is unavailable and unlikely to become available in the public domain. Hence, we used publicly available OACs as our best proxy of true cost of organs.

Given that transferring a donor to a SDCF generates an additional cost to the OPO, which will ultimately be passed on to transplant centers through the OAC, the geographic location of a SDCF may influence overhead cost. Furthermore, one limitation of this study is that our use of the $\mathrm{O}: \mathrm{E}$ ratio as a measure of performance is nuanced and potentially reflective of the performance of accepting transplant centers in close proximity to the OPO. For example, our local OPO (MOMA) transfers $31 \%$ of donors to the SDCF by plane and the remainder by ground, which may not be representative of other OPOs (4); this is particularly relevant for countries that share organs across international lines, a model adopted in Europe (11). Furthermore, given that no other OPOs had fully implemented a SDCF during the study period, our results are extrapolated from the performance of a single SDCF, which may have overstated or understated certain aspects of cost or effectiveness. However, with four other OPOs having adopted the SDCF model since 2014 and seven others in the process of doing so, it appears that the U.S. healthcare system is already aligning itself with the most cost-effective model of donor care. As more SDCFs are implemented across the country and their data becomes available, studies will be undertaken which examine the performance of multiple centers using this model.

In conclusion, we have found that from a U.S. transplant center's perspective the SDCF is a less costly, more effective model of donor care, particularly with regard to thoracic organs, when compared to the conventional model of hospital-based donor care. Our study predicts that nationwide adoption of the SDCF would result in a slightly higher number of organ transplants while substantially lowering the cost of organ acquisition. We urge international policymakers, payers, and transplant centers to consider incentives that facilitate a transition to the SDCF model of donor care.

\section{Acknowledgments}

Funding: This work was supported by NIH grants F32 HL143950 (JMG), R01 HL146856-01A1 (VP), and R21 DK110530 (SHC) and Mid-America Transplant Foundation grant 022017 (VP, SHC). The funding organizations did not directly contribute to the identification, design, conduct, or reporting of the analysis.

\section{Footnote}

Reporting Checklist: The authors have completed the 
CHEERS reporting checklist. Available at http://dx.doi. org/10.21037/jtd-20-1575

Peer Review File: Available at http://dx.doi.org/10.21037/jtd20-1575

Conflicts of Interest: All authors have completed the ICMJE uniform disclosure form (available at http://dx.doi. org/10.21037/jtd-20-1575). JMG reports grants from NIH, during the conduct of the study. MBBD reports personal fees from Novartis, outside the submitted work. WCC reports personal fees from Novartis, Pathfinder, and MidAmerican Transplant, outside of the submitted work. DK serves as an unpaid editorial board member of fournal of Thoracic Disease from Mar 2019 to Feb 2021, reports grants and personal fees from Compass Therapeutics, outside the submitted work, and has a patent pending entitled "Compositions and methods for detecting CCR2 receptors" (application number 15/611,577). SHC and VP report grants from NIH and Mid-America Transplant Foundation, during the conduct of the study. The other authors have no conflicts of interest to declare.

Ethical Statement: The authors are accountable for all aspects of the work in ensuring that questions related to the accuracy or integrity of any part of the work are appropriately investigated and resolved.

Open Access Statement: This is an Open Access article distributed in accordance with the Creative Commons Attribution-NonCommercial-NoDerivs 4.0 International License (CC BY-NC-ND 4.0), which permits the noncommercial replication and distribution of the article with the strict proviso that no changes or edits are made and the original work is properly cited (including links to both the formal publication through the relevant DOI and the license). See: https://creativecommons.org/licenses/by-nc-nd/4.0/.

\section{References}

1. United Network for Organ Sharing. News. Available online: https://unos.org/news/organ-transplants-inunited-states-set-sixth-consecutive-record-in-2018/. Accessed February 7, 2019.
2. Englesbe MJ, Merion RM. The riskiest job in medicine: transplant surgeons and organ procurement travel. Am J Transplant 2009;9:2406-15.

3. Doyle MB, Vachharajani N, Wellen JR, et al. A novel organ donor facility: a decade of experience with liver donors. Am J Transplant 2014;14:615-20.

4. Doyle M, Subramanian V, Vachharajani N, et al. Organ Donor Recovery Performed at an Organ Procurement Organization-Based Facility Is an Effective Way to Minimize Organ Recovery Costs and Increase Organ Yield. J Am Coll Surg 2016;222:591-600.

5. Chang SH, Kreisel D, Marklin GF, et al. Lung Focused Resuscitation at a Specialized Donor Care Facility Improves Lung Procurement Rates. Ann Thorac Surg 2018;105:1531-6.

6. Scientific Registry of Transplant Recipients. OPO-Specific Reports. Available online: https://www.srtr.org/reportstools/opo-specific-reports/. Accessed November 10, 2017.

7. Organ Procurement and Transplantation Network. National Data. Available online: https://optn.transplant. hrsa.gov/data/. Accessed November 10, 2017.

8. Puri V, Hachem RR, Frye CC, et al. Unintended consequences of changes to lung allocation policy. Am J Transplant 2019;19:2164-7.

9. Lindemann J, Dageforde LA, Brockmeier D, et al. Organ procurement center allows for daytime liver transplantation with less resource utilization: May address burnout, pipeline, and safety for field of transplantation. Am J Transplant 2019;19:1296-304.

10. Lynch RJ, Mathur AK, Hundley JC, et al. Improving organ procurement practices in Michigan. Am J Transplant 2009;9:2416-23.

11. Jochmans I, van Rosmalen M, Pirenne J, et al. Adult Liver Allocation in Eurotransplant. Transplantation 2017;101:1542-50.

Cite this article as: Gauthier JM, Doyle MBM, Chapman WC, Marklin G, Witt CA, Trulock EP, Byers DE, Hachem RR, Pasque MK, Meyers BF, Patterson GA, Nava RG, Kozower BD, Kreisel D, Chang SH, Puri V. Economic evaluation of the specialized donor care facility for thoracic organ donor management. J Thorac Dis 2020;12(10):5709-5717. doi: 10.21037/ jtd-20-1575 


\title{
Supplementary
}

\author{
Abbreviations \\ * $y$ : organ of interest (heart, liver, lung, kidney, or pancreas); \\ * OBS: observed; \\ * EXP: expected; \\ * PRED: predicted; \\ * $\Sigma$ : total; \\ * $\bar{x}$ : average; \\ * TXP: transplants; \\ * TXP/100: transplants per 100 donors.
}

\section{A. Data}

The following data were obtained from the SRTR reports (5): organ-specific observed transplants ( $y$ OBS TXP), number of organ donors, local and export number of transplants $\left(y \mathrm{TXP}_{\text {local }}, y \mathrm{TXP}\right.$ export $)$, and organ-specific observed-to-expected ratios (O:E) for each OPO. The local and export organ-specific OACs for each OPO was obtained from OPO-reported data from the year 2014.

\section{B. Observed organ transplants}

Organ-specific observed total transplants nationwide ( $y$ OBS $\Sigma$ TXP nationwide) was obtained by summing $y$ OBS $\Sigma$ TXP for $\mathrm{OPO}_{i}, i=1, \ldots, n$ over all OPOs.

$$
\text { yOBS } \sum \text { TXP nationwide }=\sum_{i=1}^{n} y O B S T X P \text { for } O P O_{i}
$$

where $i$ is the index for OPO, and $n$ is the number of all OPOs.

Overall observed total transplants nationwide was computed by summing yOBS $\Sigma$ TXP nationwide over all interested organs:

$$
\text { overall OBS } \sum \text { TXP nationwide }=\frac{\sum_{y} y O B S \sum T X P \text { nationwide }}{\sum_{y}}
$$

where $y$ is organ of interest: heart, liver, lung, kidney, or pancreas.

The organ-specific observed transplants per 100 donors for $\mathrm{OPO}_{i}$ is obtained by

$$
y O B S T X P / 100 \text { for } O P O_{i}=\frac{y O B S T X P \text { for } O P O_{i}}{\text { number of organ donors for } O P O_{i}} \times 100
$$

Organ-specific observed total transplants nationwide per 100 donors is computed as follows:

$$
y O B S T X P / 100 \text { nationwide }=\sum_{i=1}^{n} y O B S T X P / 100 \text { for } O P O_{i}
$$

Overall observed total transplants nationwide per 100 donors was computed by summing $y$ OBS $\Sigma T X P / 100$ nationwide over all interested organs:

$$
\text { overall OBS } \sum T X P / 100 \text { nationwide }=\frac{\sum_{y} y O B S \sum T X P / 100 \text { nationwide }}{\sum_{y}}
$$




\section{Observed costs}

Organ-specific $\mathrm{OAC}$ for $\mathrm{OPO}_{i}$ was obtained by the weighted average of local $y \mathrm{OAC}$ and export $y \mathrm{OAC}$, where weights were determined by the number of local transplants and the number of export transplants:

$$
y O A C \text { for } O P O_{i}=y \mathrm{OAC}_{i}^{\text {local }} \times \frac{y T X P_{i}^{\text {local }}}{y T X P_{i}^{\text {local }}+y T X P_{i}^{\text {export }}}+y \mathrm{OAC}_{i}^{\text {export }} \times \frac{y T X P_{i}^{\text {export }}}{y T X P_{i}^{\text {local }}+y T X P_{i}^{\text {export }}}
$$

Organ-specific observed average OAC was computed by:

$$
y O B S \bar{x} O A C=\frac{\sum_{i=1}^{n} y O A C_{i}}{n}
$$

Overall observed average OAC was computed by:

$$
\text { overall } O B S \bar{x} O A C=\frac{\sum_{y} y O B S \bar{x} O A C}{\sum_{y}}
$$

Organ-specific observed cost ( $y$ OBS $\Sigma$ cost) for each OPO was computed by multiplying the local OAC $\left(y \mathrm{OAC}^{\text {local }}\right)$ by the number of local $y$ transplants and adding this to the product of export OAC ( $y \mathrm{OAC}^{\text {Export }}$ ) and the number of export $y$ transplants.

$y O B S$ costs for $O P O_{i}=y \mathrm{OAC}_{i}^{\text {local }} \times y T X P_{i}^{\text {local }}+y \mathrm{OAC}_{i}^{\text {export }} \times y T X P_{i}^{\text {export }}$

Organ-specific observed total costs nationwide were then obtained by

$y O B S \sum$ costs nationwide $=\sum_{i=1}^{n} y O B S$ costs for $O P O_{i}$

Overall observed total costs nationwide were obtained by summing organ-specific observed total costs nationwide over all interested organs, as follows:

overall OBS $\sum$ costs nationwide $=\sum_{y} y O B S \sum$ costs nationwide

\section{Predicted organ transplants assuming nationwide adoption of the SDCF model}

Assuming nationwide adoption of the SDCF model, $y\left(\frac{O}{E}\right)_{S D C F}$ will be used as the effectiveness of each OPO, while organspecific expected total transplants for each OPO remains the same. The organ-specific predicted transplants $(y$ PRED $\Sigma T X P)$ for each OPO was calculated by multiplying the organ-specific expected total transplants ( $y$ EXP $\Sigma T X P)$ for each OPO by the SDCF's O:E ratio for that organ $\left[y\left(\frac{O}{E}\right)_{S D C F}\right]$.

$$
y \text { PREDTXP for } O P O_{i}=y E X P T X P \text { for } O P O_{i} \times y\left(\frac{O}{E}\right)_{S D C F}
$$

Organ-specific predicted total transplants nationwide ( $y$ PRED $\Sigma$ TXP nationwide) was computed by summing $y$ PRED $\Sigma$ TXP for each OPO over all OPOs.

$$
\text { yPRED } \sum \text { TXP nationwide }=\sum_{i=1}^{n} y P R E D \text { TXP for OPO }
$$


Overall predicted total transplants nationwide was calculated by summing $y$ PRED $\Sigma$ TXP nationwide over all interested organs, as follows:

overall PRED $\sum T X P$ nationwide $=\sum_{y} y P R E D \sum T X P$ nationwide

Organ-specific predicted transplants per 100 donors for each OPO was obtained by

$y$ PREDTXP $/ 100$ for $\mathrm{OPO}_{i}=\frac{y P R E D T X P \text { for } O P O_{i}}{\text { number of organ donors for } O P O_{i}} \times 100$

The organ-specific predicted total transplants nationwide per 100 donors ( $y$ PRED TXP/100 nationwide) was computed by.

yPRED $\sum T X P / 100$ nationwide $=\sum_{i=1}^{n} y P R E D T X P / 100$ for $O P O_{i}$

Overall predicted total transplants nationwide per 100 donors was calculated by summing $y$ PRED TXP/100 nationwide over all interested organs, as follows:

overall PRED $\sum T X P / 100$ nationwide $=\sum_{y} y P R E D \sum T X P / 100$ nationwide

/100 nationwide

\section{E. Predicted costs assuming nationwide adoption of the SDCF model}

Organ-specific predicted average OAC was computed by:

$y P R E D \bar{x} O A C=\frac{\sum_{i=1}^{n} y P R E D O A C \text { for } O P O_{i}}{n}$

where

$y P R E D O A C$ for $O P O_{i}=y \mathrm{OAC}_{S D C F}^{\text {local }} \times \frac{y T X P_{S D C F}^{\text {local }}}{y T X P_{S D C F}^{\text {local }}+y T X P_{S D C F}^{\text {export }}}+y \mathrm{OAC}_{S D C F}^{\text {export }} \times \frac{y T X P_{S D C F}^{\text {export }}}{y T X P_{S D C F}^{\text {local }}+y T X P_{S D C F}^{\text {export }}}$

Therefore, $y P R E D \bar{x} O A C=y P R E D \bar{x} O A C_{S D C F}$.

Overall predicted average OAC for each OPO was computed by taking the average of $y$ PRED $\bar{x}$ OAC over all interested organs.

overall PRED $\bar{x} O A C=\frac{\sum_{y} y P R E D \bar{x} O A C}{\sum_{y}}$

Organ-specific predicted costs for each OPO were obtained by:

$y$ PRED costs for $\mathrm{OPO}_{i}=y$ PREDTXP for $O P O_{i} \times y$ PREDOAC for $O P O_{i}$

Organ-specific predicted total costs nationwide were then computed by:

$y P R E D \sum$ costs nationwide $=\sum_{i=1}^{n} y P R E D$ costs for $O P O_{i}$ 


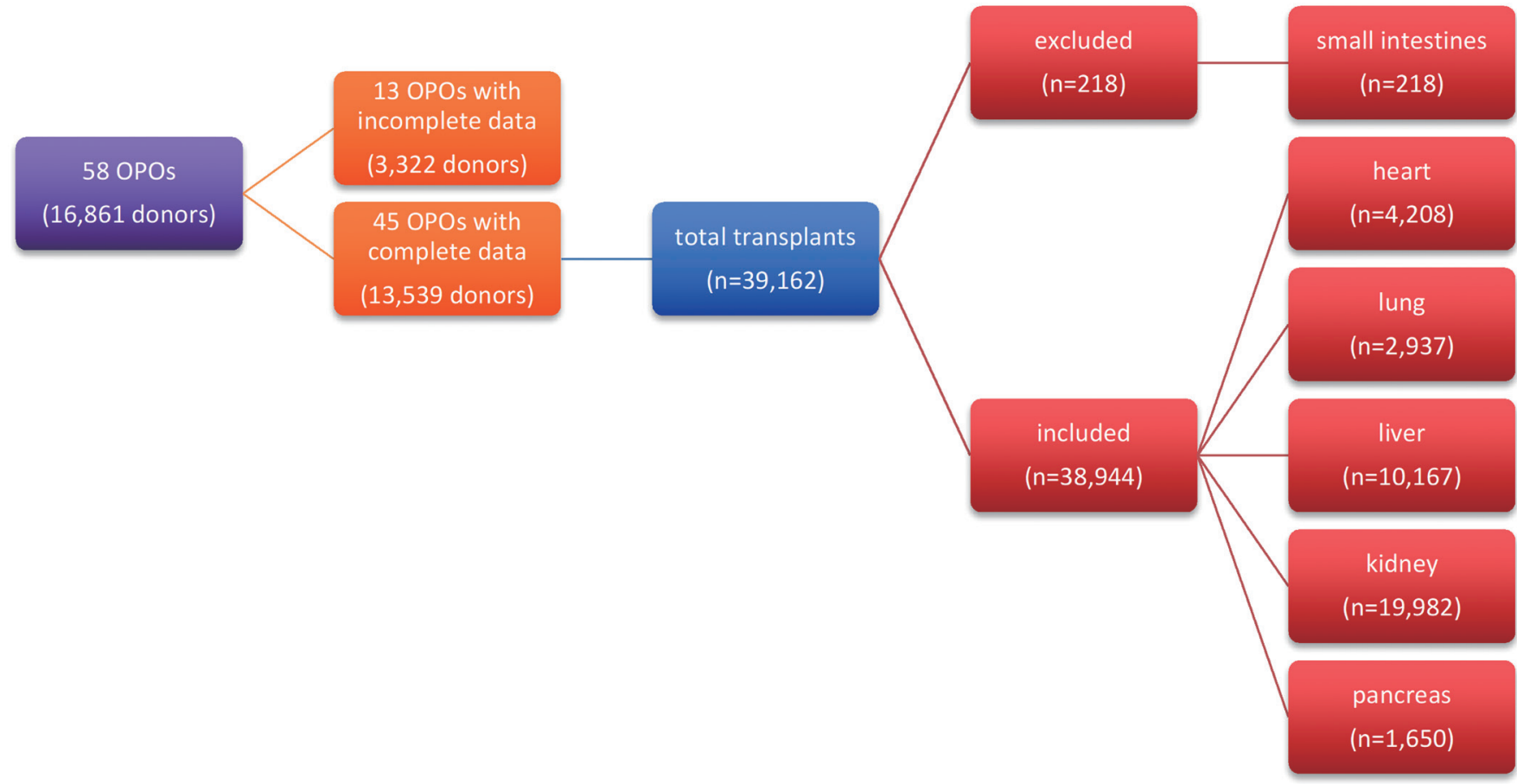

Figure S1 Flow diagram of OPOs and transplants included in the study. Of the 58 OPOs in the U.S., 45 had complete cost and effectiveness data for the 2-year study period. Due to their rarity, small bowel transplants were excluded from the study. Single and double lung transplants are counted as one organ, while kidney transplants are counted separately. Data taken from SRTR reports (5). OPOs, organ procurement organizations; SRTR, Scientific Registry of Transplant Recipients. 
Table S1 Predictors of organ yield

Age effect for brain dead donors

Blood type

Body mass index

Cardiac arrest after brain death

Cause of death

Circumstance of death

Clinical infection: blood

Clinical infection: lung

Clinical infection: other

Clinical infection: urine

Controlled DCD donor

Current cigarette use

Current cocaine use

Current other drug use

Ejection fraction (percent)

Ethnicity

Gender

Heavy alcohol use

Height

History of cancer

History of cocaine use

History of diabetes

History of hypertension

History of insulin dependence

History of other drug use

Intercept

Mechanism of death

More than 20 pack years

PHS increased infectious risk

$\mathrm{pO}_{2} / \mathrm{FiO}_{2}$ ratio

$\mathrm{pO}_{2}$

Previous Ml

Protein in urine

Race

Terminal serum creatinine

Weight

History of any diabetes

Recovered outside the contiguous 48 states?

Donor characteristics used by the Scientific Registry of Transplant Recipients to determine expected organ yield for each OPO based on the number of potential organ donors. OPO, organ procurement organization; DCD, donation after cardiac death; PHS, U.S. Public Health Service. 